\title{
Auditory frequency discrimination learning is affected by stimulus variability
}

\author{
SYGAL AMITAY, DAVID J. C. HAWKEY, and DAVID R. MOORE \\ MRC Institute of Hearing Research, Nottingham, England
}

\begin{abstract}
We explored the effects of training set variability on learning and generalization of pure-tone frequency discrimination (FD) in three groups of untrained, normally hearing adult listeners. Group 1 trained using a fixed standard tone at $1 \mathrm{kHz}$ (fixed), Group 2 on slightly varying (roving) tones around $1 \mathrm{kHz}$, and Group 3 on widely varying standard frequencies (wide-roving). Initially, two thirds of all listeners had low FD thresholds (good listeners) and one third had intermediate to high thresholds (poor listeners). For good listeners, slight variations in the training set slowed learning but wide variations did not. Transfer to untrained frequencies (up to $4 \mathrm{kHz}$ ) and to the fixed condition was equivalent regardless of training condition, but Group 1 listeners did not fully transfer learning to the roving condition. For poor listeners, any variation in the training condition slowed learning and impaired transfer to other frequencies but did not affect transfer to untrained conditions. Thus, the effects of training set on progress and outcome depend on set variability and individual FD ability.
\end{abstract}

The study of perceptual learning (see Gibson, 1953, 1963, and Goldstone, 1998, for definitions and reviews) may have great applicability; various forms of auditory discrimination training contribute to improving children's language abilities (Merzenich et al., 1996; Tallal et al., 1996) and reading skills (Kujala et al., 2001) and to reducing the severity of tinnitus (Flor, Hoffmann, Struve, \& Diesch, 2004). However, although the list of training benefits is rapidly expanding, the variables and mechanisms affecting auditory learning are still poorly understood.

Discrimination of a frequency difference between two sequentially presented pure tones has been the most extensively used task in laboratory studies of auditory perceptual learning, perhaps because consistent and robust improvement has been seen in every study (Delhommeau, Micheyl, Jouvent, \& Collet, 2002; Demany, 1985; Demany \& Semal, 2002; Grimault, Micheyl, Carlyon, Bacon, \& Collet, 2003; Grimault, Micheyl, Carlyon, \& Collet, 2002; Irvine, Martin, Klimkeit, \& Smith, 2000). Other tasks, such as sound lateralization (Wright \& Fitzgerald, 2001) and temporal-interval discrimination (Karmarkar \& Buonomano, 2003; Wright, Buonomano, Mahncke, \& Merzenich, 1997), have also been used. The common approach has been to pretest listeners on several conditions of interest, provide training on a single condition, and then retest on the original set of conditions to check for improvement. Improvement on untrained conditions is considered evi-

We thank Jabulani Sithole and Silvia Cirstea for assistance with statistical analysis and Quentin Summerfield for helpful comments on an earlier version of this article. The research was entirely supported by the Medical Research Council (U.K.). Correspondence relating to this article may be sent to S. Amitay, Developmental Cognitive Neuroscience Unit, University College London-Institute of Child Health, 30 Guilford Street, London WC1N 1EH, England (e-mail: s.amitay@ich.ucl.ac.uk). dence for generalization, or transfer, of learning. Conclusions regarding the type and underlying mechanisms of learning are then drawn from the pattern of transfer. Thus, Demany (1985) showed that frequency discrimination (FD) learning transfers to untrained frequencies within the range mediated by the same mechanism (temporal coding at low frequencies, place coding at high frequencies) but that it does not transfer across frequency ranges, whereas Irvine et al. (2000) showed that a small component of learning is specific to the trained frequency, even within the same frequency range. Delhommeau et al. (2002) showed that a large part of FD learning transfers to the untrained ear and across tone durations but that a smaller component is duration- and ear-specific. These studies suggest that tone discrimination learning has separable components, each providing evidence for a locus of operation.

A second approach acknowledges that the acoustic environment is constantly changing and that tones are rarely heard in isolation. Watson, Kelly, and Wroton (1976) reviewed a number of studies of the discrimination of two sequences of ten tones in which the frequency of a single target tone varied within the sequence. After extensive training, the FD of the target tone was only slightly worse than that for the same tone presented in isolation (as in the studies above), but only when the training sequence was fixed and the target tone had the same temporal position in the sequence. However, when trial-by-trial uncertainty about the frequency, timing, or order in sequence of the target tone was introduced, performance decreased. Spiegel and Watson (1981) later showed that once particular sequences were learned one by one, randomly intermixing them did not degrade discrimination of the trained targets. This learning did not generalize, however, to untrained sequences, temporal locations, or 
frequencies. Improvement over time was attributed to "top-down" attentional and memory processes.

The FD learning literature is thus divided into two types of studies: In one, the stimuli are very simple but are informative about levels of processing and possible mechanisms. In the other, more "realistic" complex stimuli are used. The latter studies involve large memory and attentional loads, so few conclusions can be drawn about specific perceptual processes. The study reported here attempted to bridge this gap. We used simple, isolated tones as stimuli but tested how manipulating stimulus uncertainty along one dimension (frequency) affected both the learning process and generalization of FD.

Initial rapid improvement in learning experiments is usually taken as evidence of "procedural" learning (learning related to the response demands of the task), whereas the gradual and protracted improvement following that phase has been interpreted as "perceptual" or "stimulus" learning (improvement in perceptual judgments; Robinson \& Summerfield, 1996). Since procedural learning is thought to dominate early improvement, listeners are often familiarized with the test material prior to testing and then administered an (often extensive) assessment of pretraining performance (e.g., Delhommeau et al., 2002; Grimault et al., 2002; Irvine et al., 2000). However, it is possible that perceptual learning substantially contributes to early improvement. We thus decided to forgo pretesting in favor of immediate training.

It is common practice to exclude from learning experiments listeners with initial performance that is either very good or very poor (Demany, 1985; Demany \& Semal, 2002). However, the rules of learning might differ between individuals. We have therefore chosen not only to include all listeners in our results, but to recruit listeners from a wider cross-section of the population than has typically been used for such studies in order to reflect the natural variability in human performance. We believe this inclusiveness has important ramifications for the applicability and generalizability of the results.

\section{METHOD}

\section{Participants}

Thirty-nine volunteers with normal hearing ( 23 women and 16 men, 20-39 years old), were recruited from the general population using advertisements in supermarkets, post offices, and the public library. All volunteers were native English speakers with no previ- ous experience in psychoacoustic testing and were paid for their participation. All listeners had audiometric pure-tone thresholds not exceeding $20 \mathrm{~dB} \mathrm{HL}$ at octave frequencies in the range $500-4000 \mathrm{~Hz}$. The participants were assigned to three groups that were matched for age and sex (see Table 1). The groups were also matched for number of participants who played a musical instrument, since professional musicians have lower pitch discrimination thresholds than nonmusicians (Kishon-Rabin, Amir, Vexler, \& Zaltz, 2001). Because pitch discrimination is also correlated with IQ measures (Deary, 1994), we administered the Wechsler Abbreviated Scale of Intelligence (WASI; Psychological Corporation, 1999). All methods were approved by the University of Nottingham School of Psychology Ethics Committee.

\section{Stimuli and Equipment}

The stimuli used in this experiment were pairs of 100-msec tone bursts incorporating 20-msec rise-fall times shaped with a raised cosine function. The interstimulus interval was $500 \mathrm{msec}$. Stimuli were digitally generated using custom software running on a PC that also controlled the experiment. The signal waveforms were computed on each presentation using a sampling rate of $44.1 \mathrm{kHz}$ and were generated as 16-bit samples using a sound card (Darla Echo; Echo Digital Audio Corporation, Carpinteria, CA). Stimuli were presented diotically via Sennheiser HD480II headphones at a level $70 \mathrm{~dB}$ above the listener's absolute threshold in each ear measured at the frequency closest to the standard frequency being used. The listeners were tested individually in a sound-attenuating chamber and responded on a touch screen. Each trial was initiated automatically $700 \mathrm{msec}$ after a response was made to the previous trial; there was no time limit for response. Trial-by-trial feedback was visually provided on the touch screen above the response area for 500 msec during the intertrial interval.

\section{Design and Procedure}

The experiment consisted of a training phase followed by generalization posttests. Training/testing was conducted over six sessions, each lasting approximately $1 \mathrm{~h}$. In the first session, the audiometric screen was administered, followed by the first training block of 500 trials. The listeners were told they would hear a pair of tones and were instructed to press an area on the touch screen marked " 1 " if the first tone was higher than the second and an area marked " 2 " if the second tone was higher. To ensure their understanding of the concept of high/low, the listeners were asked to choose which of two tones of different pitches vocalized by the experimenter was higher in pitch. All listeners indicated they understood the task before testing commenced. Over the next three sessions, six more training blocks were administered, two per session, with a rest interval between blocks. The fifth session consisted of a test of generalization of learning (see below) to other frequencies. The WASI test was also administered in this session. Generalization of learning across task conditions was assessed using two further blocks of testing administered in the sixth and final session. Sessions $1-5$ were typically conducted over consecutive days (exclud-

Table 1

Descriptive Statistics and Comparisons of the Three Experimental Groups

\begin{tabular}{|c|c|c|c|c|c|c|c|}
\hline & \multirow{2}{*}{$\begin{array}{l}\text { Number of } \\
\text { Listeners }\end{array}$} & \multirow{2}{*}{$\begin{array}{c}\text { Gender } \\
\text { (Females/Males) }\end{array}$} & \multicolumn{2}{|c|}{ Age (years) } & \multirow{2}{*}{$\begin{array}{c}\text { Musical } \\
\text { Experience* }\end{array}$} & \multicolumn{2}{|c|}{ Full-Scale IQ $^{* *}$} \\
\hline & & & $M \pm S D$ & Range & & $M \pm S D$ & Range \\
\hline Group 1: Fixed & 12 & $5 / 7$ & $30.7 \pm 7.0$ & $20-39$ & 5 & $123.8 \pm 9.2$ & $102-137$ \\
\hline Group 2: Roving & 12 & $8 / 4$ & $29.1 \pm 6.1$ & $21-38$ & 7 & $119.9 \pm 10.4$ & $103-139$ \\
\hline Group 3: Wide roving & 15 & $10 / 5$ & $29.2 \pm 6.5$ & $20-39$ & 8 & $118.1 \pm 11.2$ & $99-137$ \\
\hline$p$ & & $.34^{\dagger}$ & .80 & & $.70^{\dagger}$ & $.36 \ddagger$ & \\
\hline
\end{tabular}

Note - Group names refer to the type of training received and are explained in the "Training conditions" subsection. *Number of listeners who play a musical instrument. ${ }^{* *}$ Wechsler Abbreviated Scale of Intelligence (Psychological Corporation, 1999). †Pearson chi-square test. ॠOne-way ANOVA. 
ing weekends), but occasionally additional days (not exceeding 7) intervened. The sixth session was administered after a variable period (up to 3 months from the commencement of testing).

In each phase of the experiment, difference limens for frequency (DLFs), expressed as the percentage of the standard frequency, were estimated using an adaptive two-interval, two-alternative forced choice paradigm. For each trial, one interval contained the standard tone and one contained a comparison tone of higher frequency. The interval containing the comparison tone was chosen randomly in each trial. The listener's task was to detect the interval containing the comparison tone.

In all DLF assessments, a two-down, one-up rule was used to estimate the frequency difference corresponding to the $70.7 \%$ correct point on the psychometric function (Levitt, 1971). The initial difference in frequency was $20 \%$ of the standard frequency; the difference was reduced after two consecutive correct responses and was increased after one incorrect response using a three-tier stepsize design. A step size of $4 \%$ was used until 7 reversals had occurred, a $1 \%$ step size was used during a further 4 reversals, and $0.2 \%$ was used until a total of 100 trials had elapsed, at which point the adaptive procedure was terminated. The threshold (or DLF) was taken as the arithmetic mean of the frequency differences at all of the reversal points from the 12th onward (an average of 18 reversals, or 55 trials, per DLF). Ten percent of all trials were "easy," designed to assess the listeners' attentiveness to the task; the frequency difference between the standard and comparison tones on these trials was $5 \%$ above the frequency difference on the previous adaptive trial. These trials did not affect the progress of the adaptive procedure and were not included in calculating the DLF.

Training conditions. In the training phase (Sessions 1-5), the listeners received extensive FD training on one of three conditions:

1. Fixed. The standard-tone frequency was fixed at $1000 \mathrm{~Hz}$.

2. Roving. The standard-tone frequency was varied around $1 \mathrm{kHz}$ $(900,950,1000,1050$, and $1100 \mathrm{~Hz})$.

3. Wide-roving. The standard tone was varied at frequencies outside the $1-\mathrm{kHz}$ critical band, and frequencies were separated by two critical bandwidths according to the Bark scale (570, 840, 1170, 1600, and $2150 \mathrm{~Hz}$ ).

Twelve listeners were trained on the fixed condition (Group 1), 12 on the roving condition (Group 2), and 15 on the wide-roving condition (Group 3). All listeners were trained over seven blocks. Each block consisted of five independent, interleaved adaptive tracks (either five tracks at $1 \mathrm{kHz}$ in the fixed condition or one track per frequency in the roving and wide-roving conditions). The track that controlled stimulus level was pseudorandomly selected for each trial in the block, ensuring that 100 trials were presented from each of the adaptive tracks.

Posttraining tests. Following the training sessions, two kinds of posttests were administered, in this order:

1. Generalization of learning across frequencies. All listeners in Groups 1 and 2, as well as 3 listeners from Group 3, were administered a single-track DLF estimation at the following standard frequencies: $1000,2000,4000$, and $500 \mathrm{~Hz}$, tested in that order. The other 12 listeners in Group 3 were administered only three DLF estimates, at 2000, 4000, and $500 \mathrm{~Hz}$, to avoid exposure to $1000 \mathrm{~Hz}$. This test was administered in the fifth session.

2. Transfer of learning across task conditions. The listeners in Group 1 were administered two blocks of the roving condition, and the listeners in Groups 2 and 3 were administered two blocks of the fixed condition. This test was administered in the sixth session. Two Group 2 listeners did not return to complete this posttest, and data from one block for a Group 3 listener were lost as a result of computer failure.

Threshold estimates and missing data. Each track yielded a single DLF estimate. In a preliminary analysis, we found that these estimates did not differ across multiple tracks in any of the conditions, whether the standard tone was fixed or varied across tracks.
Thus, the DLF estimates from the five tracks were geometrically averaged. Error rates on the easy trials interspersed among the adaptive track trials were generally low (Group 1, 1.9\%; Group 2, $5.0 \%$; Group 3, 7.0\%) and did not differ significantly between training groups [one-way analysis of variance (ANOVA): $F(2,36)=$ $1.77, p=.19]$.

Data were missing for 13 of the total 1,365 training runs (39 listeners $\times 7$ training blocks $\times 5$ tracks per block). In Group 1, not enough reversals had occurred in 3 instances, so threshold estimates could not be generated. One listener in Group 2 completed only 6 of the 7 training blocks, and as mentioned earlier the data from one block for one Group 3 listener were lost as a result of a technical problem. We filled the missing values using an expectation maximization algorithm (SPSS, Version 11; SPSS, Chicago, IL). These values were not included in the figures and were used only for the purpose of statistical analysis.

\section{RESULTS}

\section{Training}

The training data for each group, expressed as DLF thresholds, are shown in Figure 1. Both individual data (thin lines) and group geometric means (thick lines) are shown. In all three training groups, initial performance was highly variable, ranging over two orders of magnitude. About two thirds of the listeners in each group had good FD (DLFs smaller than 1\%; solid thin lines), and a third performed more poorly (DLFs from $2 \%$ to over 20\%; dotted thin lines). Listeners in Group 1 with poor initial performance improved very quickly and dramatically, but those in Groups 2 and 3 showed more gradual improvement and had persistently high thresholds throughout the training phase. Group means were strongly influenced by unusually high DLFs. We therefore divided the listeners into subgroups based on performance at the start of training (Block 1), with two thirds of the listeners in each group designated "good" and one third "poor" (first-block DLFs under $2 \%$ and over $2 \%$, respectively). The best listeners in Group 1 had DLFs between $0.2 \%$ and $0.4 \%(2-4 \mathrm{~Hz})$ at $1 \mathrm{kHz}$, in close agreement with observed DLFs for highly trained listeners in other psychoacoustic studies (e.g., Wier, Jesteadt, \& Green, 1977).

Training data are shown separately for the good and poor listeners in each training group in Figures $2 \mathrm{~A}$ and 2B, respectively. The good listeners in Group 2 showed more gradual learning and higher thresholds in comparison with those in Groups 1 and 3 [training group, $F(2,23)=$ $9.31, p=.001$; block $\times$ group interaction, $F(12,138)=$ $2.09, p=.034]$. On the other hand, the poor listeners in both Groups 2 and 3 showed more gradual learning and higher thresholds in comparison with those in Group 1 [training group, $F(2,10)=4.92, p=.033$; block $\times$ group interaction, $F(12,60)=3.36, p=.001]$.

\section{Posttest 1: Generalization of Learning Across Frequencies}

Posttraining performance of each group on a series of fixed frequencies is shown in Figure 3. Good listeners (Figure 3A) had lower thresholds than poor listeners 

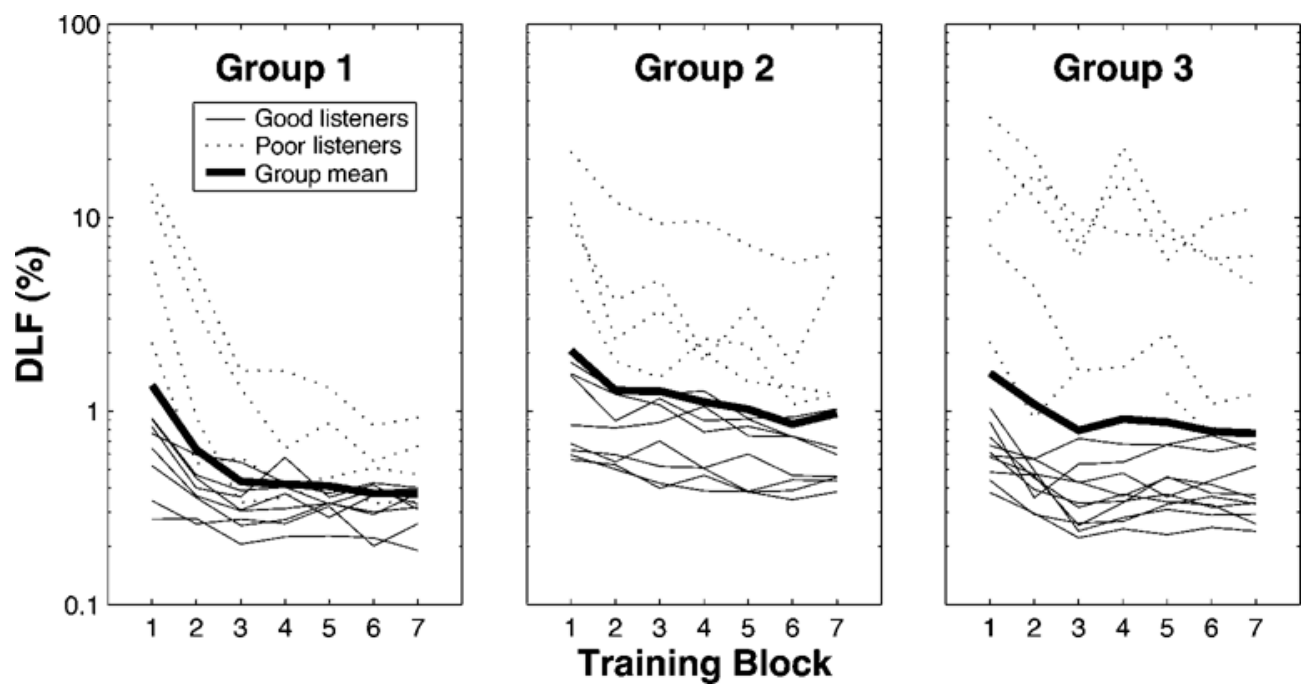

Figure 1. Individual training data for good (thin solid lines) and poor (thin dotted lines) listeners and group mean (thick lines), expressed as frequency difference limens (DLF).

(Figure 3B) overall, but training condition had no effect on DLFs for fixed $1-\mathrm{kHz}$ tones [good, $t(14)=-1.49$, $p=.16$; poor, $t(6)=-0.85, p=.43]$. Only 3 listeners in Group 3 were tested with $1-\mathrm{kHz}$ tones; their data are shown in Figure 3 only as individual points, and they were excluded from the analysis.

At the untrained frequencies, all good listeners had equivalent FD thresholds, regardless of training group [repeated measures ANOVA: $F(2,21)=0.73, p=.49$ ]; poor listeners in Group 1 had marginally lower thresholds than did those in Groups 2 and $3[F(2,9)=4.14$, $p=.053]$. DLFs did not differ across frequencies [good, $F(2,42)=1.95, p=.16$; poor, $F(2,18)=0.15, p=.82]$.

\section{Posttest 2: Transfer of Learning Across} Task Conditions

Figure 4 shows the results for the second posttest, which compared performance of each training group on the trained and untrained conditions. Group 2 and Group 3 listeners performed the fixed condition as well as those listeners trained on it (Group 1; Figures 4A, 4B) in both the good [repeated measures ANOVA: $F(2,20)=0.53$, $p=.57]$ and poor $[F(2,8)=0.94, p=.43]$ subgroups. This is especially remarkable in the case of Group 3 listeners, who were not exposed to FD around $1 \mathrm{kHz}$ prior to this test. No learning was observed between the two test blocks in either good or poor listeners.
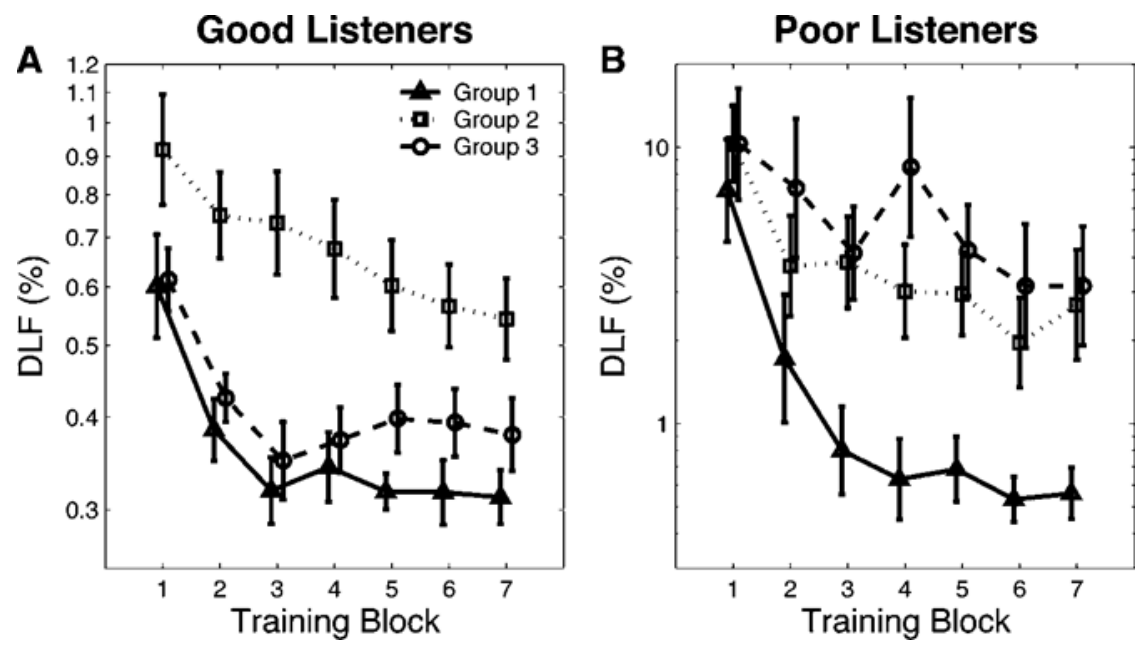

Figure 2. Mean frequency difference limens (DLF) over the seven training blocks for good (A) and poor (B) listeners in Groups 1 (triangles), 2 (squares), and 3 (circles). Error bars represent standard errors of the mean. 

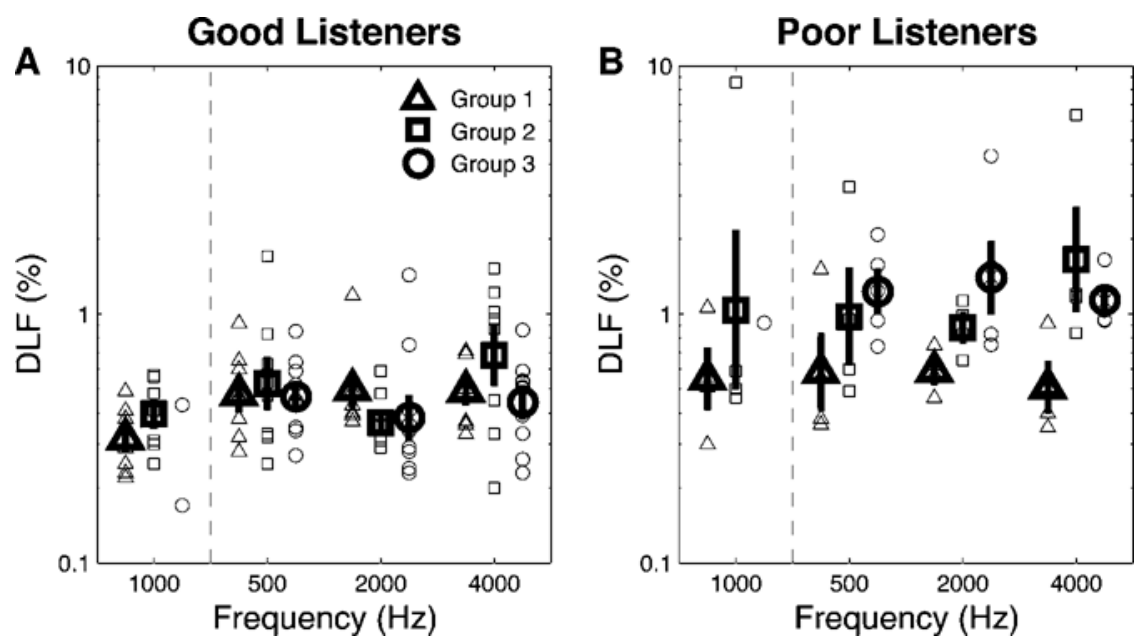

Figure 3. Generalization from the trained frequency to other frequencies (Posttest 1) in frequency difference limens (DLF). Group mean (large symbols) and individual data points (small symbols) for good (A) and poor (B) listeners in Groups 1 (triangles), 2 (squares), and 3 (circles). Error bars represent standard errors of the mean.
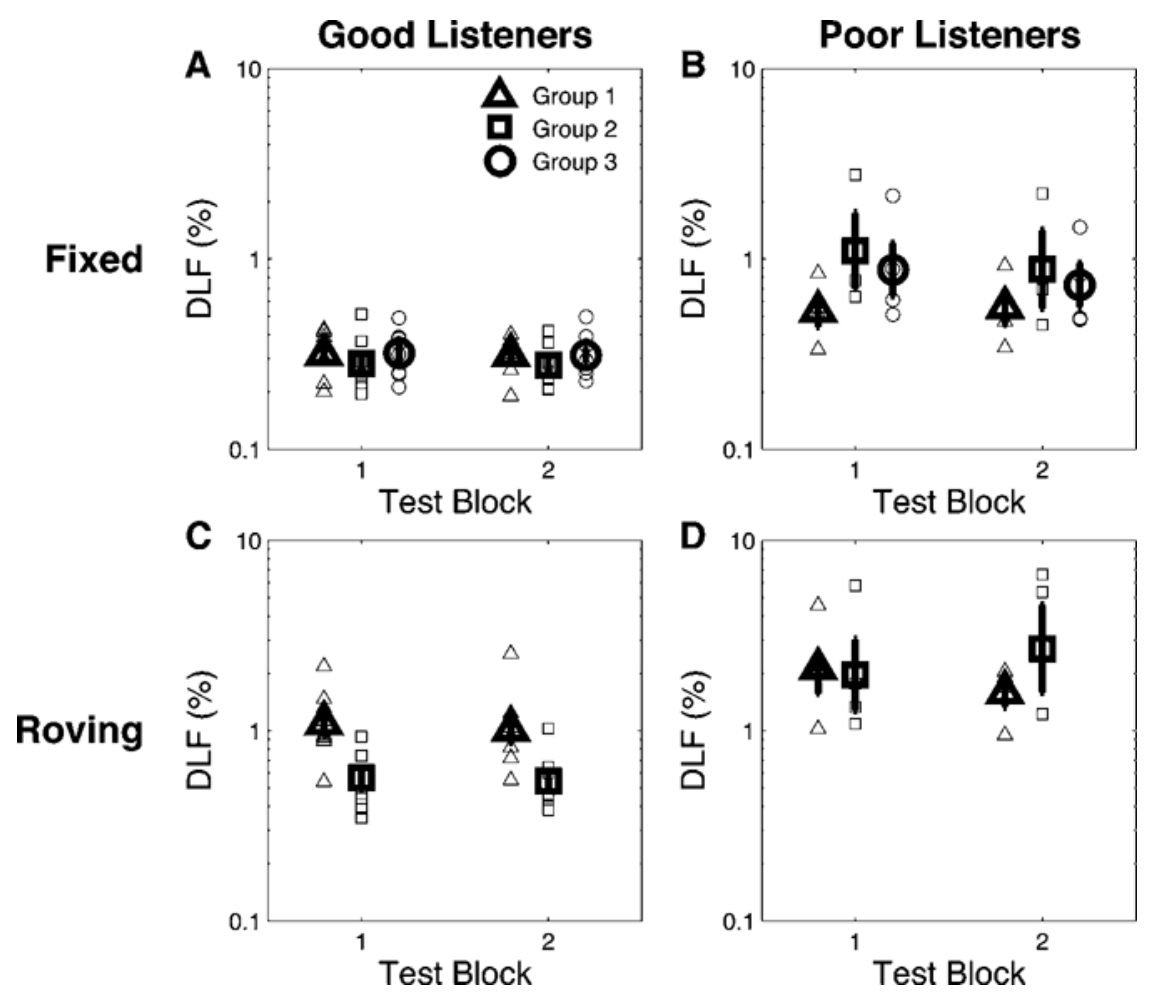

Figure 4. Generalization from the trained condition to other test conditions (Posttest 2) in frequency difference limens (DLF). (A, B) Comparison of group performance on the fixed condition: Group means (large symbols) and individual data points (small symbols) for good (A) and poor (B) listeners in Groups 1 (triangles), 2 (squares), and 3 (circles). Data for Group 1 are taken from the 6th and 7th training blocks (see Figure 2). (C, D) Comparison of group performance on the roving test condition for good (C) and poor (D) listeners. Data for Group 2 are taken from the 6th and 7th training blocks (see Figure 2). Error bars represent standard errors of the mean. 
Good listeners (Figure 4C) trained on the roving condition (Group 2) had lower DLFs in this condition after training than did those trained on the fixed condition (Group 1) [repeated measures ANOVA: $F(1,13)=10.5$, $p=.006]$. In fact, good listeners in Group 1 were not better on the roving condition than Group 2 listeners were on their first training block (see Figure 2A). Poor listeners (Figure 4D) had higher DLFs than good listeners but did not differ according to training condition $[F(1,6)=0.24, p=.64]$.

\section{Overview of Main Findings}

The main finding in this study was that learning of FD depends on an interaction between individual perceptual capabilities and the variability of the training set.

In Group 1, improvement was commensurate with initial performance: The higher the initial DLF, the greater the observed improvement. Good listeners in Groups 1 and 3 started well and showed further early improvements. Initially poor Group 1 listeners showed rapid and dramatic improvement over the first three blocks $(1,500$ trials) of training. Over the next four blocks their thresholds were virtually unchanged. All Group 2 listeners and the poor listeners in Group 3 showed slower, protracted learning that appeared to be incomplete after seven blocks of 500 trials.

Good listeners had similar thresholds regardless of training condition on all generalization posttests, with one exception: Those trained on the roving condition had lower thresholds on this condition than those trained on the fixed condition. Poor listeners had consistently higher thresholds throughout the posttests, but those in Group 1 tended to have lower thresholds than those in Groups 2 and 3 .

\section{DISCUSSION}

\section{Training in Frequency Discrimination}

As previously reported, intensive training improved frequency discrimination thresholds in all listeners (Delhommeau et al., 2002; Demany, 1985; Demany \& Semal, 2002; Irvine et al., 2000). Individual variability in thresholds ranged over almost two orders of magnitude. This was unsurprising, as we did not use any performancebased selection criteria and no pretraining/testing was given (see Demany, 1985; Demany \& Semal, 2002; Irvine et al., 2000). However, it is this individual variability that appears to determine the nature and outcome of the learning process. Initial ability predicts not only the time course of learning, but also its outcome in terms of transfer to untrained frequencies and untrained conditions. Thus, individual differences are important in understanding the nature of perceptual learning.

\section{Good Listeners}

The majority of the listeners had low thresholds for FD even at the commencement of training. In these good listeners, varying the frequency reduced performance and slowed learning, but varying the frequency widely did not seem to affect either performance or learning in comparison with listeners trained on a fixed frequency. The effect of roving range thus appears to be biphasic, with surprisingly strong performance for widely variable training stimuli.

Energetic masking by preceding or subsequent tones is unlikely to be the cause of degraded perception in the roving condition, as the interstimulus and intertrial intervals were long enough to counteract conventional forward or backward masking (Elliott, 1971). Alternatively, these results may reflect an attention effect. In order to isolate a stimulus perceptually from its context, attention must be focused on the "listening band" containing it (Schlauch \& Hafter, 1991). Unlike critical bands, which are fixed in width, listening bands are under cognitive control; their width and sensitivity, and even the number of bands a listener monitors at any one time, can be altered by task demands. Two listening strategies are possible when detecting tones of uncertain frequency: One can shift attention from one narrow listening band to another, thereby preserving resolution but increasing the attentional load, and hence the difficulty in detection (Green, 1958). Alternatively, one can widen a single band to include all the frequencies being monitored, thereby reducing attentional load but also impairing resolution. In either case, the obvious prediction is that detection will deteriorate with increasing separation of the frequencies used (Creelman, 1960; Veniar, 1958a, 1958b). However, cues can improve detection of uncertain frequencies to the level of detection of fixed frequencies (Johnson \& Hafter, 1980; Swets \& Sewall, 1961). The cue may serve to decrease the attentional load involved in monitoring multiple bands and shifting attention. Thus, it may only be effective when using the attentional shift strategy. In our two-interval discrimination experiment, both tones were clearly audible, and the first tone may have acted as a cue to the listening band position. In the wide-roving condition, in which the listening bands were widely separated, the first tone in the pair may have directed attention immediately to the correct band. In the roving condition, however, adjacent frequencies may have been too close to make the second listening strategy effective. This may, in turn, have both eliminated the cuing effect and impaired resolution relative to the wideroving and fixed conditions, respectively. If this argument is true, it can explain the apparent discrepancy between our results and those of Jesteadt and Bilger (1974). They found that increasing the frequency-roving range progressively decreased performance on an FD task; "jittering" the frequency over $40 \mathrm{~Hz}$ did not impair performance, but varying it over $465 \mathrm{~Hz}$ (roving) did. However, the frequencies in their jittered condition were close enough together that the band did not need to be widened by much, and resolution did not suffer. In their roving condition, the frequencies were far enough apart that the width of the listening band seriously hampered resolution, as also happened in our roving condition. 
In support of this argument, research in vision has shown that shape discrimination of items of uncertain position in visual arrays is inhibited in proportion to their spatial proximity (the visual equivalent of spectral proximity) to an attentionally salient stimulus (Mounts, 2000a, 2000b). Disengaging and shifting attention from an attention-capturing element was easier when the target element was farther away from it, even though the distance over which attention had to be shifted was greater.

We suggest that Group 2 listeners trained on both FD (the purely "perceptual" aspect of the task) and an additional, attentional aspect. The latter may have involved either a gradual improvement in resolution within a wide listening band or a move from listening on a wide band to increasingly separated overlapping bands (change of listening strategy). Perceptual learning of FD proceeded normally alongside this attentional component, as evidenced by complete transfer to the fixed condition and other frequencies. Since this attentional component was absent in the fixed condition, Group 1 listeners did not perform the roving condition as well as those trained on it. We predict that training on wide-roving frequencies would not transfer completely to the roving condition, either. Unfortunately, we did not test Group 3 listeners on the roving condition, and thus cannot confirm this prediction.

The results from Posttest 2 were similar to those that have been obtained for speech sound training, in that listeners trained on a limited set of phonetic contrasts did not perform as well on a more variable posttest set as did listeners trained on the variable set (Lively, Logan, \& Pisoni, 1993). This suggests that speech sound training and training on simple auditory stimuli may share the same general learning rules.

Exposure to a particular frequency is not necessary for learning. Listeners trained on the wide-roving condition were not exposed to FD around $1 \mathrm{kHz}$ prior to the condition generalization (Posttest 2), yet they had the same threshold on the fixed condition as the two groups who were trained at $1 \mathrm{kHz}$. Moreover, discrimination of frequencies from 0.5 to $4 \mathrm{kHz}$ was the same and constant for all good listeners, regardless of the condition on which they trained. Other studies have shown various degrees of pitch specificity in FD training (Demany, 1985; Demany \& Semal, 2002; Irvine et al., 2000). Although we did not measure generalization directly (by comparing performance before and after training), the similarity of thresholds at different frequencies supports full transfer of learning to untrained frequencies (up to $4 \mathrm{kHz}$ ).

\section{Poor Listeners}

Introducing stimulus uncertainty impaired performance and slowed learning for the poor listeners. The difference between good and poor listeners in Group 3 is most striking. It is possible that the attention demands of monitoring multiple listening bands are too high for the poor listeners, or that they are unable to use effectively the cues provided by the first tone in every pair. In the roving condition, they may use the same strategy as the good listeners, but in the wide-roving condition, they may find shifting attention between listening bands difficult. What distinguishes good from poor listeners may therefore be the listening strategies they employ (see Penner, 1972; Swets \& Kristofferson, 1970), which are limited by the attention resources at their disposal. In any case, it is clear that the listening strategy can improve with training, as poor listeners showed improvement even when trained with a variable-frequency training set.

\section{CONCLUSION}

The majority of our sample listeners had good FD and performed poorly only when the frequency varied slightly between trials. Perceptual learning of FD occurred similarly in all good listeners. Conversely, in poorer listeners, those who trained without variability in the stimulus set showed an advantage, and such training resulted in substantial improvement both for trained and untrained stimuli. This divergence between good and poor listeners suggests that learning may differ between individuals. If the results we found are generalizable to other forms of learning, training programs may benefit from an initial assessment of performance on the trained task, with subsequent expansion or restriction of training materials for better or poorer performers, respectively.

Finally, this study provides evidence for early perceptual learning. Performance on the different training conditions diverged from the very first block. Since the differences between conditions affected only the perception of the stimuli, we suggest that perceptual learning makes a greater contribution to early performance improvement than has previously been thought. Furthermore, more recent data from our lab support this conclusion (Hawkey, Amitay, \& Moore, 2004). We found that by using various perceptual judgment or response paradigms during training and observing further learning on a target task sharing either perceptual or response demands with the training task, perceptual learning accounted for most of the early performance improvement.

\section{REFERENCES}

Creelman, C. D. (1960). Detection of signals of uncertain frequency. Journal of the Acoustical Society of America, 32, 805-810.

DEARY, I. J. (1994). Intelligence and auditory discrimination: Separating processing speed and fidelity of stimulus representation. Intelligence, 18, 189-213.

Delhommeau, K., Micheyl, C., Jouvent, R., \& Collet, L. (2002). Transfer of learning across durations and ears in auditory frequency discrimination. Perception \& Psychophysics, 64, 426-436.

Demany, L. (1985). Perceptual learning in frequency discrimination. Journal of the Acoustical Society of America, 78, 1118-1120.

Demany, L., \& Semal, C. (2002). Learning to perceive pitch differences. Journal of the Acoustical Society of America, 111, 1377-1388.

Elliott, L. L. (1971). Backward and forward masking. Audiology, 10, 65-76.

Flor, H., Hoffmann, D., Struve, M., \& Diesch, E. (2004). Auditory discrimination training for the treatment of tinnitus. Applied Psychophysiology \& Biofeedback, 29, 113-120.

GIBSON, E. J. (1953). Improvement in perceptual judgments as a func- 
tion of controlled practice or training. Psychological Bulletin, 50, 401-431.

Gibson, E. J. (1963). Perceptual learning. Annual Review of Psychology, 14, 29-56.

Goldstone, R. L. (1998). Perceptual learning. Annual Review of Psychology, 49, 585-612.

GREEN, D. M. (1958). Detection of multiple component signals in noise. Journal of the Acoustical Society of America, 30, 904-911.

Grimault, N., Micheyl, C., Carlyon, R. P., Bacon, S. P., \& Collet, L. (2003). Learning in discrimination of frequency or modulation rate: Generalization to fundamental frequency discrimination. Hearing Research, 184, 41-50.

Grimault, N., Micheyl, C., Carlyon, R. P., \& Collet, L. (2002). Evidence for two pitch encoding mechanisms using a selective auditory training paradigm. Perception \& Psychophysics, 64, 189-197.

HaWkey, D. J. C., Amitay, S., \& Moore, D. R. (2004). Early and rapid perceptual learning. Nature Neuroscience, 7, 1055-1056.

Irvine, D. R. F., Martin, R. L., Klimkeit, E., \& Smith, R. (2000). Specificity of perceptual learning in a frequency discrimination task. Journal of the Acoustical Society of America, 108, 2964-2968.

JeSTEADT, W., \& BILGER, R. C. (1974). Intensity and frequency discrimination in one- and two-interval paradigms. Journal of the Acoustical Society of America, 55, 1266-1276.

Johnson, D. M., \& HAFTER, E. R. (1980). Uncertain-frequency detection: Cuing and condition of observation. Perception \& Psychophysics, 28, 143-149.

Karmarkar, U. R., \& Buonomano, D. V. (2003). Temporal specificity of perceptual learning in an auditory discrimination task. Learning \& Memory, 10, 141-147.

Kishon-Rabin, L., Amir, O., VeXler, Y., \& Zaltz, Y. (2001). Pitch discrimination: Are professional musicians better than non-musicians? Journal of Basic \& Clinical Physiology \& Pharmacology, 12, 125-143.

Kujala, T., Karma, K., Ceponiene, R., Belitz, S., Turkkila, P., TerVANIEMI, M., \& NÄÄTÄNEN, R. (2001). Plastic neural changes and reading improvement caused by audiovisual training in readingimpaired children. Proceedings of the National Academy of Sciences, 98, 10509-10514.

LEVITT, H. (1971). Transformed up-down methods in psychoacoustics. Journal of the Acoustical Society of America, 49, 467-477.

Lively, S. E., Logan, J. S., \& Pisoni, D. B. (1993). Training Japanese listeners to identify English /r/ and /l/: II. The role of phonetic environment and talker variability in learning new perceptual categories. Journal of the Acoustical Society of America, 94, 1242-1255.

Merzenich, M. M., Jenkins, W. M., Johnston, P., Schreiner, C., Miller, S. L., \& Tallal, P. (1996). Temporal processing deficits of language-learning impaired children ameliorated by training. Science, 271, 77-81.

MounTs, J. R. (2000a). Attentional capture by abrupt onsets and feature singletons produces inhibitory surrounds. Perception \& Psychophysics, 62, 1485-1493.
MounTs, J. R. (2000b). Evidence for suppressive mechanisms in attentional selection: Feature singletons produce inhibitory surrounds. Perception \& Psychophysics, 62, 969-983.

Penner, M. J. (1972). The effect of payoffs and cue tones on detection of sinusoids of uncertain frequency. Perception \& Psychophysics, 11, 198-202.

Psychological CoRporation (1999). WASI: Wechsler Abbreviated Scale of Intelligence. San Antonio: Harcourt Brace.

Robinson, K., \& SuMMERFIELD, A. Q. (1996). Adult auditory learning and training. Ear \& Hearing, 17, 51S-65S.

SCHLAUCH, R. S., \& HAFTER, E. R. (1991). Listening bandwidths and frequency uncertainty in pure-tone signal detection. Journal of the Acoustical Society of America, 90, 1332-1339.

SPiegel, M. F., \& WATSON, C. S. (1981). Factors in the discrimination of tonal patterns: III. Frequency discrimination with components of well-learned patterns. Journal of the Acoustical Society of America, 69, 223-230.

Swets, J. A., \& Kristofferson, A. B. (1970). Attention. Annual Review of Psychology, 21, 339-366.

SweTs, J. A., \& SEwall, S. T. (1961). Stimulus vs. response uncertainty in recognition. Journal of the Acoustical Society of America, 33, 1586-1592.

Tallal, P., Miller, S. L., Bedi, G., Byma, G., Wang, X., Nagarajan, S. S., Schreiner, C., Jenkins, W. M., \& Merzenich, M. M. (1996). Language comprehension in language-learning impaired children improved with acoustically modified speech. Science, 271, 81-84.

VENIAR, F. A. (1958a). Signal detection as a function of frequency ensemble: I. Journal of the Acoustical Society of America, 30, 10201024.

VENIAR, F. A. (1958b). Signal detection as a function of frequency ensemble: II. Journal of the Acoustical Society of America, 30, 10751078 .

Watson, C. S., Kelly, W. J., \& Wroton, H. W. (1976). Factors in the discrimination of tonal patterns: II. Selective attention and learning under various levels of stimulus uncertainty. Journal of the Acoustical Society of America, 60, 1176-1186.

Wier, C. C., Jesteadt, W., \& Green, D. M. (1977). Frequency discrimination as a function of frequency and sensation level. Journal of the Acoustical Society of America, 61, 178-184.

Wright, B. A., Buonomano, D. V., Mahncke, H. W., \& Merzenich, M. M. (1997). Learning and generalization of auditory temporalinterval discrimination in humans. Journal of Neuroscience, 17, 3956-3963.

Wright, B. A., \& Fitzgerald, M. B. (2001). Different patterns of human discrimination learning for two interaural cues to soundsource location. Proceedings of the National Academy of Sciences, 98, 12307-12312.

(Manuscript received March 3, 2004;

revision accepted for publication September 7, 2004.) 